\title{
Desarrollo rural y reforma agraria en Colombia: ¿prosperidad para todos?
}

\section{Kyla Sankey}

\begin{abstract}
La necesidad de reforma agraria y desarrollo rural ha estado en la agenda nacional de Colombia durante casi un siglo, y sigue vigente hoy en dia. Sin embargo, el sistema económico nacional y mundial, así como la función y el alcance del Estado, han sido sometidos a transformaciones extremas desde los últimos grandes intentos de reforma agraria en los años sesenta. En este contexto, se analizan los actuales proyectos en la materia.
\end{abstract}

INTRODUCCIÓN

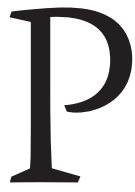
ara el sociólogo y periodista Alfredo Molano, en la larga historia conflictiva de Colombia, «el origen del problema — siempre tapado a balaes la tierra». ${ }^{1}$ No cabe duda que con la mitad de la población sumida en la pobreza, un desplazamiento interno de cinco millones de personas y $52 \%$ de la tierra en manos de $1.1 \%$ de la población, los programas de reforma agraria y desarrollo rural son clave para la resolución de conflictos sociales en el ámbito rural del país. No es casual que el tema de desarrollo rural integral sea el primer punto en las actuales negociaciones de paz entre las Fuerzas Armadas Revolucionarias de Colombia (FARC) y el gobierno. Sin embargo, el consenso se acaba en cuanto a la interpretación de la cuestión agraria y los programas de desarrollo rural y reforma agraria.

En enero de 2012, entró en vigencia el proyecto que supuestamente le permitirá al presidente Juan Manuel Santos dejar su huella en la historia: la Ley de Víctimas y Restitución de Tierras, misma que pretende devolver tres millones de hectáreas de tierra a campesinos desplazados, además de impulsar proyectos de «desarrollo rural integral». Esta ley ha sido acompañada por un discurso que inspira grandes esperanzas: según el ministro de Agricultura y Desarrollo Rural, es «tan o más importante» que el primer intento de reforma agraria de los años treinta, $y$ «más ambiciosa» que el segundo intento de reforma agraria bajo la Alianza para el Progreso en los sesenta. ${ }^{2}$ En el mismo tono, el presidente Santos ha 
declarado que la restitución de tierras le quita la razón de ser a la guerrilla y que generará un desarrollo «integral» que traerá «prosperidad para todos». Por cierto, la meta es ambiciosa: se propone resolver el problema de la pobreza rural en Colombia con una solución a la que, hasta ahora, el gremio de terratenientes más poderoso del país no se ha opuesto.

Aparte de la retórica elevada, las promesas del gobierno son reveladoras de la práctica del desarrollo hoy en día: se propone resolver los conflictos sociales más antiguos del país mediante escenarios de ganar-ganar (win-win); proyectos de desarrollo y una reforma agraria que pretenden beneficiar a todos. «Esta revolución agraria no es entre los ricos y entre los pobres, esto no es una lucha de clases», afirma Santos. ${ }^{3}$ La comparación que hace el ministro de Agricultura con los dos programas de reforma agraria del siglo pasado es pertinente: los dos surgieron tras movilizaciones rurales que exigían cambios radicales; el primero tras la formación de ligas campesinas en los años veinte y treinta, y el segundo después de un ola de tomas de tierra en los cincuenta y sesenta, en un contexto internacional marcado por el éxito de la Revolución cubana. Ambos proyectos eran percibidos como una amenaza al poder de las clases de terratenientes y empresarios, y terminaron siendo abortados frente a la resistencia de los gremios representantes de estos grupos. En cambio, el proyecto actual llega tras dos décadas de represión paramilitar que ha arroja- do el asesinato de miles de líderes de sindicatos, grupos campesinos, indígenas, afrodescendientes y partidos de oposición. Pese a la continuidad de un importante movimiento rural, los derechos de propiedad privada de las clases dominantes no están amenazados.

¿Cuáles son las circunstancias que han llevado a estos cambios? ¿Cuál es el motivo del más reciente programa de desarrollo rural? En un primer momento, este artículo examina las diferencias en el alcance de los proyectos colombianos de reforma agraria y desarrollo de los años sesenta, y los de hoy en día. El argumento central es que, en el discurso dominante, los términos han sido cambiados para favorecer los intereses del gran capital en colaboración con las clases dominantes del país, no sólo con respecto a lo que se puede lograr con el desarrollo y la reforma agraria, sino también acerca de la capacidad del Estado para realizar intervenciones a gran escala favorables a la población en general y sobre lo que los ciudadanos pueden exigir de sus gobiernos. Estos cambios son el producto de un proyecto que busca imponer una ideología y un modelo de desarrollo con el apoyo de mecanismos de represión y exclusión política.

En ese contexto, la presentación del desarrollo rural y la reforma agraria, como asuntos que pueden lograrse mediante procesos neutrales y técnicos que traen beneficios para todos, debe entenderse como el resultado de un proyecto intencional de redefinición del alcance del desarrollo y del papel del Estado en los intereses del gran capital y las clases dominantes. Hoy en día, se per-

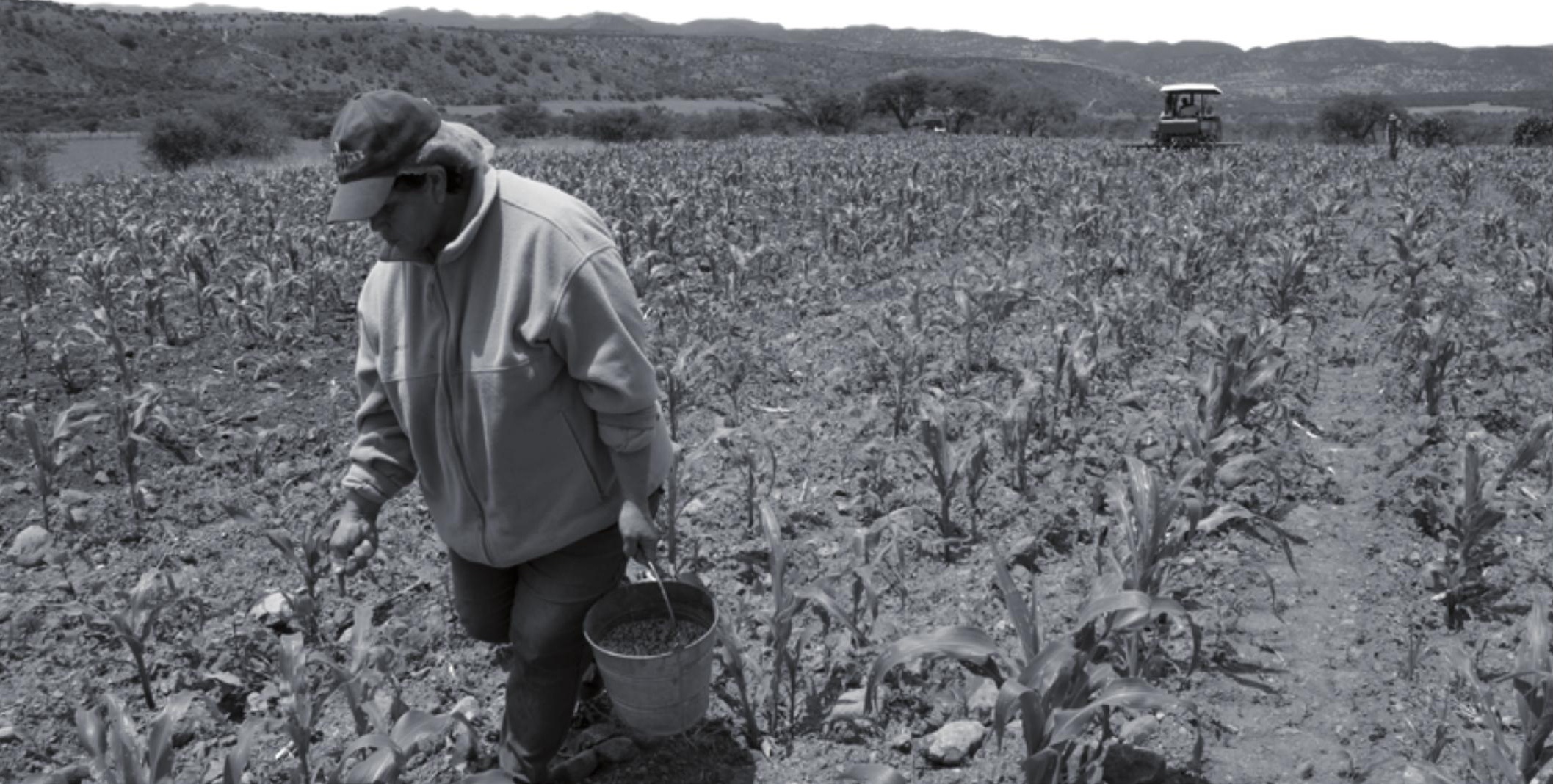


filan dos tipos de proyectos de desarrollo: mientras que los proyectos de gran escala buscan consolidar procesos que agudizan las desigualdades, los proyectos de desarrollo local rural y reforma agraria buscan mitigar los impactos de descomposición social y desviar exigencias para cambios más radicales. Por tanto, son mejor entendidos como una especie de «hoja de parra ideológica». ${ }^{4}$

El impacto de los proyectos era de corto alcance, sólo se distribuyó 10\% del millón de hectáreas programado; la mayoría de las tierras que se venderian eran las más improductivas $y$ no contaban con infraestructura adecuada.
(BM), bajo el liderazgo de Eduardo $\mathrm{Cu}$ rrie. Los economistas del BM promovían un modelo de «desarrollo económico acelerado», que no dependía de los pequeños agricultores sino de un proceso de acumulación basado en la construcción de monopolios de agronegocios, consolidado por el levantamiento de tarifas nacionales y regionales, y la minimización de otros «obstáculos» al proceso de acumulación, tales como los pro-

\section{EL SIGNIFICADO DE LA REFORMA}

\section{AGRARIA Y EL DESARROLLO}

RURAL, AYER Y HOY

El último intento de emprender un proyecto de reforma agraria desde el Estado ocurrió en casi todo América Latina en los años sesenta bajo la Alianza para el Progreso. En el contexto del reciente éxito de la Revolución cubana, la Alianza buscó ofrecer una alternativa capitalista a un cambio revolucionario. Esta «alternativa» se basaba en los modelos de desarrollo keynesianos de los economistas de la Comisión Económica para América Latina y el Caribe (CEPAL) que buscaban transformar las relaciones rurales «pre-capitalistas» e improductivas. La modernización dependía de la capacidad del Estado «activista» o «desarrollista» para intervenir, regular y planificar la economía nacional con la meta de remplazar las importaciones y generar un mercado interno. El modelo se basaba en la capacidad productiva del pequeño agricultor, entendido como el principal abastecedor de alimentos para la población nacional. Por lo tanto, la reforma agraria era necesaria para expropiar las tierras latifundistas improductivas con el fin de emprender una redistribución de parcelas pequeñas bajo títulos colectivos o individuales. Una agenda de desarrollo nacional dependía de la capacidad del Estado de realizar programas de reforma agraria y desarrollo rural a gran escala.

En Colombia, el programa de reforma agraria, bajo la Alianza, se enfrentó con una fuerte resistencia de los cabildeos de terratenientes y sus aliados en el Congreso. Tras varios intentos de bloquear la redistribución limitada que se había logrado, finalmente, en el Pacto de Chicoral de 1972, se concretó el abandono de la reforma agraria. ${ }^{5} \mathrm{El}$ rechazo del modelo venía acompañado con una ideología alternativa, una visión de desarrollo promocionada por un grupo de economistas neoclásicos del Banco Mundial gramas de bienestar. En esta visión, un componente integral del progreso económico y social era el éxodo de los pequeños productores «atrasados», quienes proveerían una abundante fuerza de trabajo barato para las nuevas industrias urbanas y rurales. ${ }^{6}$ De acuerdo con el modelo, el proyecto de reforma agraria de la Alianza fue reemplazado en 1976 por el Programa de Desarrollo Rural Integrado (DRI), financiado por el BM y el Banco Interamericano de Desarrollo (BID). Este programa apostaba por los procesos del mercado y dio prioridad a los productores con mayor capacidad de acumulación e integración al mercado, con asistencia en forma de crédito y tecnología. La introducción de nuevas variedades de semillas, pesticidas y fertilizantes aumentó los costos de producción en 575\%.?

La adopción del modelo allanó el camino para las restructuraciones neoliberales de los años ochenta y, especialmente, noventa, con la implementación de las políticas de apertura y ajustes estructurales de conformidad con el Consenso de Washington. Con la desregulación financiera, los mercados nacionales fueron expuestos a los flujos de capital, lo cual, en conjunto con la especulación de la moneda nacional, llevó a la desestabilización de la planificación macroeconómica. Las restricciones de la política monetaria del Banco Central, reforzadas por las condiciones impuestas por el Fondo Monetario Internacional (FMI), a cambio de un préstamo de 2.7 mil millones de dólares, resultaron en una supuesta «incapacidad» del Estado para realizar inversiones productivas a gran escala. En este marco, el principal papel estatal en el ámbito rural fue promover un modelo que busca integrar la agricultura a las cadenas globales de mercancías para ganar acceso a la inversión directa extranjera y facilitar los pagos de la deuda externa a través de exportaciones que cuentan con «ventajas comparativas» a nivel internacional.

Las transformaciones rurales de aquella época crearon las condiciones que hoy en día dominan el cam- 
po colombiano. Al tener que competir con los agricultores estadounidenses, quienes recibieron 62 veces más subsidios que los colombianos, entre 1980 y 1998 la producción agrícola se redujo de $43 \%$ a 13\% del producto interno bruto (PIB), y las importaciones aumentaron de 215 millones de dólares a 715 millones de dólares durante los años noventa. ${ }^{8}$ Hoy en día, se importa 10 millones de toneladas de alimentos, lo cual equivale a la pérdida de un millón de puestos de trabajo. La correspondiente reducción en productos nacionales (maíz, trigo) fue remplazado con ganadería, y el gobierno reaccionó a la crisis de producción con un impulso hacia productos de exportación «estratégicos»: soya, azúcar y palma aceitera. ${ }^{9}$ El desplazamiento masivo no fue acompañado con un aumento en la oferta de empleos: la tasa de desempleo aumentó de $10.8 \%$ en 1991 a $20.4 \%$ en $2000 .{ }^{10}$ En el contexto de estas políticas, el cambio en la concentración de tierra es revelador: en 1994, 34\% de la tierra estaba en manos de cinco mil terratenientes y en 2001 la cifra llegó a 48\%. Además, entre 1997 y 2000, la incidencia de pobreza creció de $50 \%$ a $60 \%$ de la población. ${ }^{11}$ En general, el nuevo modelo de desarrollo generó una gran transferencia de riqueza, de los más pobres a los más ricos de la sociedad.

Cabe destacar que un componente integral de la implementación del nuevo modelo de desarrollo era la violencia que dominaba las relaciones sociales. Dentro del modelo de «desarrollo económico acelerado», la militarización y paramilitarización de la sociedad tuvo tres resultados que favorecen al capital. Primero, ayudó a mantener los costos de la fuerza de trabajo a un nivel bajo mediante una fuerte represión de los sindicatos. Según la Central Unitaria de Trabajadores, desde 1984, más de 2,800 sindicalistas han sido asesinados; $49 \%$ por los paramilitares y $43 \%$ por los agentes del Estado. Hoy en día, la tasa de sindicalización es menor a $5 \% .{ }^{12} \mathrm{Se}$ gundo, aceleró el proceso histórico de acumulación por desposesión. Se calcula que desde 1985, 5.2 millones de campesinos han sido desplazados de $10 \mathrm{mi}$ llones de hectáreas, ${ }^{13} \mathrm{y}$ varios casos han demostrado que se usa la tierra despojada para los proyectos de agronegocios o de minería a gran escala; en ambos sectores los vínculos paramilitares han sido comprobados. ${ }^{14}$ Finalmente, aseguró la exclusión de toda voz política que cuestionaba el modelo dominante. La Unión Patriótica, el partido político de la izquierda que surgió a finales de los años ochenta, vía negociaciones con las Fuerzas Armadas Revolucionarias de Colombia-Ejército del Pueblo (FARC-EP), terminó con el asesinato de cuatro mil miembros, incluso alcaldes, congresistas, senadores y candidatos presidenciales. En Colombia, al igual que en varios de los países vecinos, el lema de Margaret Thatcher sobre el neoliberalismo - «no hay alternativas» - toma un tono más siniestro: se eliminan las alternativas a través de un programa de violencia perpetua.

Con el apoyo de la represión política, durante la introducción del modelo neoliberal se logró establecer dentro del discurso dominante un cambio en el significado de desarrollo y de reforma agraria. Mientras que, entre los años cincuenta y setenta, los términos se referían a la idea del desarrollismo asociado con la CEPAL, en los años ochenta se redefinió «desarrollo» como participación en el mercado global. De acuerdo con la nueva definición, los proyectos de desarrollo y reforma agraria tomaron una nueva dirección. Antes, para los desarrollistas, el desarrollo nacional consistía en un proyecto integral que incluía una visión de desarrollo del pequeño agricultor dentro de las metas de la macroeconomía nacional, dirigido por el Estado. En cambio, con las transformaciones impulsadas por el Consenso de Washington, la meta del desarrollo nacional se refleja en una serie de medidas que busca facilitar la inserción de la economía nacional en el mercado mundial a través de las políticas de ajustes estructurales y estabilización.

Cuando las políticas del Consenso de Washington terminaron con una amplia descomposición social, en los noventa se formuló un Posconsenso de Washington que pretende mitigar los impactos más des-
En los últimos 10 años, casi $40 \%$ del territorio colombiano ha sido concesionado a empresas mineras; de los 114 millones de hectáreas del territorio nacional, 8.4 millones han sido concesionadas para la exploración minera y más de 37 millones de hectáreas están tituladas para exploración de hidrocarburos. estabilizadores del modelo neoliberal a través de políticas microeconómicas orientadas a reducir la pobreza extrema y fortalecer la sociedad civil. El proyecto buscó cambiar lo que se entendía por desarrollo; ya no era la transformación de la sociedad sino una salida de la pobreza a nivel individual. De acuerdo con este modelo, el agente responsable de realizar cambios ya no era el Estado desarrollista sino el mercado.

Sobre esta línea, un sinfín de informes ha señalado cómo reducir la pobreza rural con medidas que ayu- 
dan al pobre a insertarse en el mercado. La meta de la reforma agraria también adquirió un nuevo significado. El agente responsable para la redistribución de la tierra era el mercado; la redistribución se lograría a través de un programa de créditos que facilitara la compra-venta voluntaria de tierras bajo el auspicio del BM. Cabe destacar que en Colombia se iniciaron las reformas en las zonas con los índices de conflicto más altos. ${ }^{15} \mathrm{El}$ impacto de los proyectos de los noventa y principios del siglo XxI era de corto alcance, sólo terminó con la distribución de apenas 10\% del millón de hectáreas programado; la mayoría de las tierras que se venderían voluntariamente eran las más improductivas y las que no contaban con sistemas de infraestructura adecuada. ${ }^{16}$

Según Brittain, la designación de «desarrollo» al nuevo proyecto es poco más que «una hoja de parra ideológica». Según él, el propósito del proyecto es «legitimar lo que eran, en realidad, esfuerzos por el capital estadounidense de penetrar mercados o de apropiar y controlar los recursos/bienes de otras naciones, y frecuentemente las dos cosas». ${ }^{17}$ Cabe resaltar que este esfuerzo del capital imperialista fue aceptado a causa de la conveniencia que tenía con las relaciones sociales ya existentes dentro del país; era una filosofía muy conveniente para las clases dominantes.

\section{PANORAMA DE HOY: \\ DESARROLLO NACIONAL}

Actualmente, dos proyectos políticos dominan el programa de desarrollo rural de Colombia. Primero, el Plan de Desarrollo Nacional 2010-2014: «Prosperidad para todos» (PND) prevé un crecimiento económico impulsado por la «locomotora minera», en otras palabras, una nueva fase de acumulación primitiva acelerada. En los últimos 10 años, casi $40 \%$ del territorio colombiano ha sido concesionado a empresas mineras; de los 114 millones de hectáreas de territorio nacional, 8.4 millones han sido concesionadas para exploración de minerales y más de 37 millones de hectáreas están tituladas para exploración de hidrocarburos. ${ }^{18}$ En cuanto a los títulos de exploración de minerales, ha habido un aumento de 3,700\% en los últimos 10 años. Una sola empresa, Anglo Gold Ashanti, tiene un millón de hectáreas concesionadas en 16 departamentos de Colombia. ${ }^{19}$ Este modelo incluye una política de supresión de regalías. Según los cálculos del investigador Mario Valencia, debido a las exenciones tributarias, el gobierno se queda con sólo $10 \%$ de las ganancias del sector minero, y si se toma en cuenta los costos ambientales y sociales, el saldo es negativo; en otras palabras, los colombianos pagan a las compañías mineras para que exploten minerales. ${ }^{20}$ Además, el boom del sector minero ha resultado en una revaluación del peso de más de 10\%, lo que reduce la competitividad de otros sectores, donde se encuentra la mayoría de los trabajadores; el sector minero cuenta con sólo 5\% del empleo del país. Esto se traduce en un deterioro en los estándares de vida para los trabajadores que no están en el sector minero. ${ }^{21}$

Segundo, una serie de políticas estatales buscan promover la expansión de los agronegocios. Colombia tiene un tratado de libre comercio (TLC) suscrito con la Unión Europea, uno vigente con Estados Unidos y otro está en negociaciones con China. Además del levantamiento de tarifas, los TLC incluyen varios mecanismos para proteger las inversiones extranjeras. En ese contexto, se facilitan los nuevos mecanismos de acaparamiento de tierras, donde especuladores o empresas extranjeras compran o arriendan predios masivos en países que tienen una «ventaja comparativa» para cultivos que atienden a las demandas globales alimentarias y energéticas, por ejemplo, maíz, soya, palma aceitera y azúcar. Actualmente, el gobierno está en proceso de abrir la última gran frontera agrícola del país: cuatro millones de hectáreas en la Altillanura Oriental. Grandes bloques de tierra están siendo concesionadas a empresas nacionales e internacionales de agroindustria y el nuevo «mercado verde» del sector forestal. ${ }^{22}$

\section{HOJAS DE PARRA}

En ese contexto surge el segundo PND que busca mitigar el impacto de la descomposición social. En cuanto a las motivaciones del proyecto detrás de
Grandes bloques de tierra están siendo concesionadas a empresas nacionales e internacionales de agroindustria $y$ el nuevo «mercado verde» del sector forestal. este plan, un informe del BM es revelador. Primero, con respeto a los TLC, el informe constata que «el levantamiento de barreras al comercio sin duda favorece el alivio de la pobreza y promueve el crecimiento», y que se requieren ajustes para que los sectores menos productivos, incluso la agricultura de pequeña escala, puedan reorientarse hacia la producción de bienes más aptos para la exportación..$^{23}$ Entre otras medidas, 
la más importante en el ámbito rural es un «despegue agrícola basado en la ventaja comparativa» que emplea «la mano de obra intensiva». ${ }^{24}$ El desarrollo depende de los agronegocios de palma, soya o flores, por ejemplo. El pequeño agricultor tiene que encontrar la manera de insertarse en el modelo, o bien desplazarse para vender su «mano de obra intensiva» a los empresarios «más competitivos». De acuerdo con el informe, el PND se compromete a fomentar «el desarrollo de grandes explotaciones», en tanto que el ministro de Agricultura señaló que las regalías se usarán "para el financiamiento de grandes emprendimientos en el campo agropecuario». ${ }^{25}$ En este contexto, es difícil prever otra consecuencia, salvo la continuación del éxodo del pequeño productor.

Según el BM, la capacidad administrativa del Estado de garantizar los derechos de propiedad es clave para aprovechar la «ventana de oportunidad». El documento consta que «el uso eficiente de la tierra requiere las condiciones que faciliten la movilidad de los derechos de propiedad, para que la tierra pueda utilizarse en las actividades más productivas y por los usuarios más eficientes». ${ }^{26}$ Para el BM, la meta de establecer títulos de propiedad claros se logrará a través de un sistema de créditos que pretende formalizar los derechos sobre 1.5 millones de hectáreas en terrenos baldíos.

En este contexto, cabe preguntar, ¿qué tipo de desarrollo rural prevé la Ley de Víctimas y Restitución de Tierras? Compuesto por 324 artículos, el documento instaura las líneas para una restitución de predios pequeños a personas desplazadas a través de un conjunto de medidas jurídicas y administrativas que establece la formalización de la propiedad rural y derechos sobre los predios rurales. Según las más recientes cifras del gobierno, actualmente se analizan 140 mil reclamos de víctimas desplazadas de más de un millón de hectáreas de tierra, pero estas cifras han sido cuestionadas por la oposición. ${ }^{27}$

En cuanto al proyecto de restitución, las dudas más fuertes son dos. Primero, a pesar de la supuesta desmovilización de los paramilitares en 2005, el campo colombiano sigue siendo vigilado por grupos paramilitares y una gran cantidad de tierras despojadas están actualmente en posesión de paramilitares que siguen activos. Eso conlleva un gran riesgo para las personas despojadas. Entre 2006 y 2011, fueron asesinados 71 líderes de comunidades que reclamaban tierras despojadas. ${ }^{28}$ Relacionado con eso, la segunda duda gira en torno a la dificultad que se prevé en el proceso de restitución, en tanto implica una confrontación entre las fuerzas del capital y las víctimas. Se calcula que la tercera parte de los predios más productivos de agronegocios son «propiedad de capitales de dudosa procedencia», ${ }^{29} \mathrm{y}$ entre el proceso de desplazamiento y la promulgación de la Ley, una gran proporción de la tierra ha sido concesionada a empresas mineras. ${ }^{30}$

En ese contexto, hay una alta probabilidad de que varias previsiones de la ley terminen con la facilitación del proceso de clarificación y formalización de derechos de propiedad y la amplificación del mercado de tierras. El artículo 99 establece la posibilidad de que el reclamante celebre contratos con las empresas que explotan el predio. También existe la posibilidad de vender el predio al Estado. Eso podrá coincidir con la creación, bajo la misma ley, de instituciones regionales y procedimientos clarificados para la concesión de extensiones ilimitadas de tierra a entidades públicas para su explotación. Dado que el mismo gobierno ha resaltado en varias ocasiones que la gran mayoría de los desplazados no quieren retornar a sus tierras, resurgen grandes dudas sobre los beneficiarios previstos en la ley.

Los alcances principales de la Ley son dos: primero, el desvío de los crecientes movimientos sociales que reclaman tierras, así como la comunidad internacional de derechos humanos; segundo, la facilitación del proceso de acumulación de tierras en manos del gran capital, vinculado con las clases dominantes nacionales. De esta manera, se abre el camino para la última fase de la acumulación neoliberal, mejor vista como la desposesión legalizada, y no como la restitución de tierras.

\section{CONCLUSión}

Las desigualdades rurales siguen vigentes y tienen la misma relevancia, o más, que en la época del desarrollismo. Asimismo, sigue siendo urgente realizar el desarrollo rural y una reforma agraria. Sin embargo, un proyecto ideológico y de represión política ha logrado establecer la hegemonía de un modelo de «desarrollo nacional» que agudiza desigualdades y extiende el modelo de acumulación por desposesión. Al mismo tiempo, los proyectos de desarrollo local o de reforma agraria buscan, por un 
lado, disminuir la oposición al modelo mediante la promoción de respuestas individuales a problemas sociales y el retiro del Estado como agente principal responsable de realizar los cambios que favorecen la mayoría de los ciudadanos; por otro lado, buscan facilitar la desposesión del campesinado por la vía legal.

Sin embargo, el establecimiento de una ideología dominante, por toda la fuerza que tenga, no es capaz de invertir las circunstancias materiales que han traído la necesidad histórica de afrontar la cuestión agraria. Lo importante es no dejar que una confusión intencional en los términos del debate desoriente la necesidad de investigar y afrontar las estructuras del capitalismo contemporáneo. Además, no debe menguar lo que se puede y debe exigir de un proyecto de desarrollo rural integral y reforma agraria.

\section{Referencias}

1 Alfredo Molano Bravo, «La tierra, siempre la tierra», Espectador, 2010. Disponible en línea 〈http://www.elespectador. com/opinion/columnistasdelimpreso/alfredo-molano-bravo/columna-205730-tierra-siempre-tierra〉.

2 Semana, «Esta ley es más ambiciosa que la de López Pumarejo», 2012a. Disponible en línea 〈http://www.semana.com/especiales/pilares-tierra/esta-ley-es-mas-ambiciosa-quela-de-lopez-pumarejo.html.

3 «Presidente Santos afirma que la revolución agraria de su Gobierno es integral». Disponible en línea 〈http://wsp.presidencia.gov.co/Prensa/2012/Enero/Paginas/20120127_04.aspx>.

4 James Brittain, «A Theory of Accelerating Rural Violence: Lauchlin Currie's Role in Underdeveloping Colombia», Journal of Peasant Studies, volumen 32, número 2, 2005, p. 336.

5 Héctor Mondragón, Colombia: o mercado de tierras o reforma agraria, 2002. Disponible en línea «ttp://www.acantioquia.org/documentos/prob_agraria/mercado_tierras_reforma_agraria.pdf».

6 Brittain, op. cit., pp. 337-342.

7 Francis Thomson, «The Agrarian Question and Violence in Colombia: Conflict and Development», Journal of Agrarian Change, volumen 11, número 3, 2011, pp. 321-356.

8 Forest Hylton, Evil hour in Colombia, Verso, Londres y Nueva York, 2006, p. 85.

9 Carlos Jaramillo, «Liberalization, Crisis, and Change: Colombian Agriculture in the 1990s», Economic Development and Cultural Change, volumen 49, número 4, 2001, pp. 821-846.

10 Eric Ross, «Clearance as Development Strategy in Rural Colombia», Peace Review, volumen 19, número 1, 2007, p. 62.

11 Mondragón, op. cit.

12 justiceforcolombia, About Colombia, 2012. Disponible en línea 〈http://justiceforcolombia.org〉.

13 ABColombia, Returning Land to Colombia's Victims, 2011, pp. 1-2. Disponible en línea «ttp://www.abcolombia.org.uk/ downloads/ReturningLandReportforweb.pdf».

14 Ibidem, p. 5.

15 Mondragón, op. cit. pp. 7-8.
16 Santurnino Borras, «Questioning Market-Led Agrarian Reform: Experiences from Brazil, Colombia and South Africa», Journal of Agrarian Change, volumen 3, número 3, 2003, pp. 381-384.

17 Brittain, op. cit. p. 336

18 PBI Colombia, Minería en Colombia: ¿a qué precio?, 2011. Disponible en línea 〈http://www.pbi-colombia.org/fileadmin/user_files/projects/colombia/files/colom PBIa/111122 boletin_final_web.pdf .

19 ABColombia, op cit.

20 рві Colombia, op. cit., p. 9

21 James Petras, Colombia: Extractive Capital and Peace Negotiations, 2012. Disponible en línea http://petras.lahaine. $\operatorname{org} /$ ? $\mathrm{p}=1910$ 〉.

22 Justicia y Paz Colombia, «Mercado de Tierras en Colombia: ¿Acaparamiento o soberaníaalimentaria?», 2012. Disponible en línea 〈http://justiciaypazcolombia.com/IMG/pdf/Cartilla_ IMCA_FINAL_FINAL_1_.pdf $>$.

23 Banco Mundial, Colombia 2006-2010: Una ventana de oportunidad, Washington, Banco Mundial, 2007, p. 14.

24 Ibidem, p. 17.

25 Jorge Robledo, Santos, Tierra y Libre Comercio, 2010. Disponible en línea http://www.argenpress.info/2010/09/santos-tierra-y-libre-comercio.html.

26 Banco Mundial, op. cit.

27 Semana, Restrepo capoteó las críticas del Polo a la restitución de tierras, 2012b. Disponible en línea 〈http://www.semana. com/politica/restrepo-capoteo-criticas-del-polo-restitucion-tierras/177981-3.aspx>.

28 Semana, Reina impunidad en asesinatos de lideres de tierras, 2012c. Disponible en línea 〈http://www.semana.com/nacion/ reina-impunidad-asesinatos-lideres-tierras/175153-3. aspx>.

29 Semana, La tierra prometida, 2011. Disponible en línea 〈http://m.semana.com/nacion/tierra-prometida/144024-3. aspx>.

30 ABColombia, op. cit. 